\title{
'|||||||||||||||||||||||||||||||||||||||||||||||||||||||||||||||||||||.
}

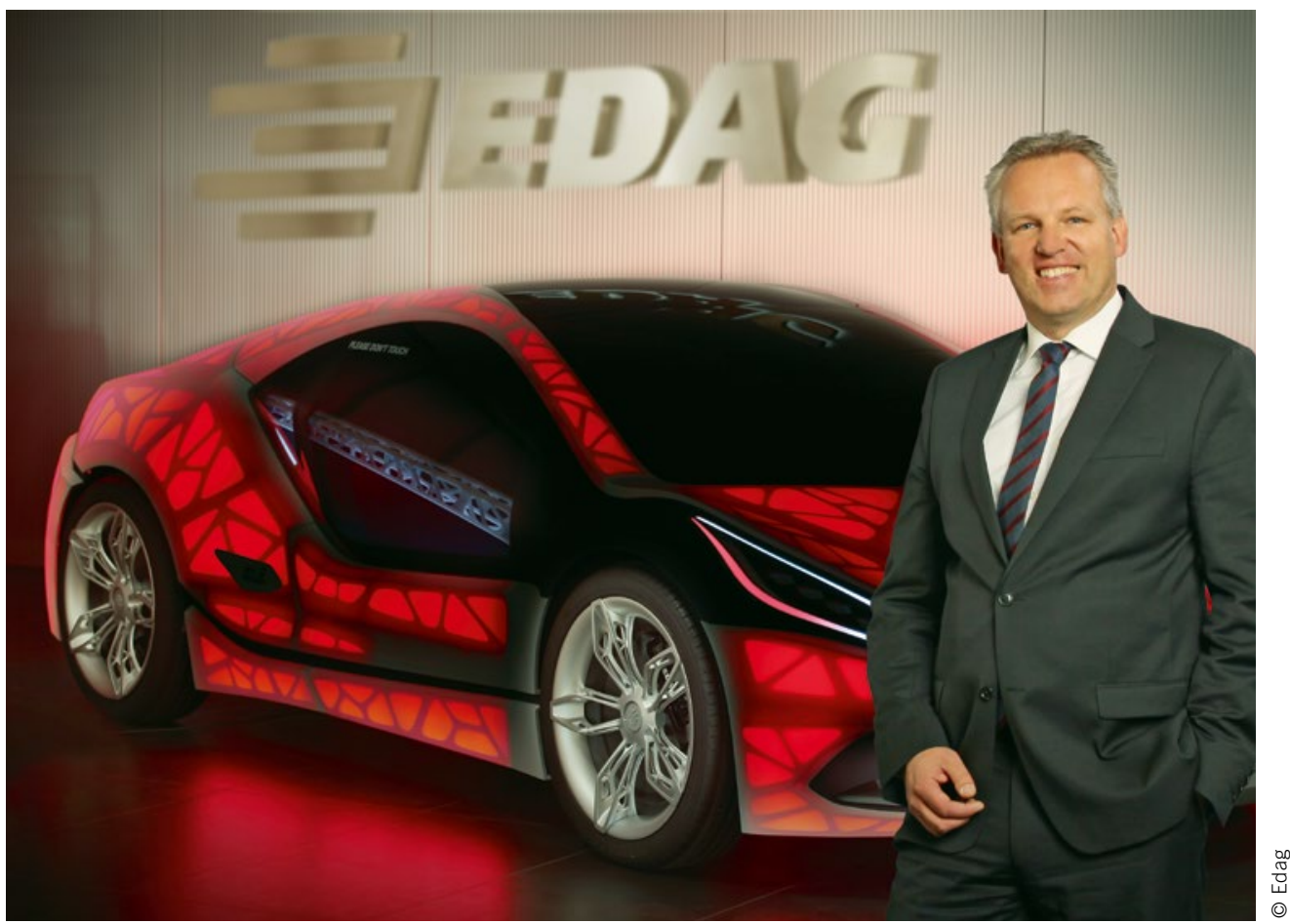

Dipl.-Ing. Jörg Ohlsen

CEO of Edag Engineering GmbH

\section{Mastering the Variety of Options}

Over the last few years, the trend towards an increasing number of vehicle types in the automotive industry has remained unchanged. Customers' demand behaviour and the growing internationalisation of the markets, with attendant localisations, have further reinforced this development. Parallel to this, the developers are faced with the need to integrate alternative drive concepts and energy storage systems. The resulting package situations have different effects on the dimensioning of the vehicle body and the distribution of weight in the complete vehicle. This means that a wide variety of adjustments is inevitable.

The consequence is an exponential increase in the challenges with regard to the flexible design of body concepts and production processes - particularly when these concepts must first be implemented in scenarios involving very small volumes. We are, of course, still called upon to continue our work on advancing lightweight design, as a means both of contributing towards sustainable products and of working out acceptable economic solutions.

It is becoming more and more difficult with current strategies to maintain the fine balance between variance, volume, cost and lightweight design. All engineering companies are required to apply lateral thinking, and identify alternative body and manufacturing processes. One promising approach we can see lies in the combination of additively manufactured parts and bionically inspired design. With the "Edag Light Cocoon" concept car, we have already introduced a vision of vehicle body production using very few tools, which also makes use of ultra-lightweight structures.

Although such extensive additive manufacturing is not yet suitable for full-scale production, we still regard partial solutions as feasible in the medium term. In cooperation with Laser-Zentrum Nord, Concept Laser and the BLM Group, for instance, we have developed a bionically optimised, hybrid manufactured spaceframe concept for a body node. Its success lies in the combination of additively produced body parts and conventionally manufactured sections. Thanks to additive manufacturing, the steel nodes can be highly flexible and multifunctional, to enable, for instance, different vehicle variants to be produced on demand in an extremely flexible production cell: without additional tooling, production equipment and start-up costs. The result is a bionically designed, load path-optimised spaceframe node.

As the process uses very little in the way of equipment and tools, it will be possible for all body variants to be produced economically and with the maximum possible flexibility in the future. We will gradually be transferring the potential of additive manufacturing to concrete applications, to give a future-proof answer to the question of mastering the variety of options. 